Virginia Commonwealth University VCU Scholars Compass

2005

\title{
Evolution of the electronic structure of Be clusters
}

\author{
V. Cerowski \\ Virginia Commonwealth University \\ B. K. Rao \\ Virginia Commonwealth University \\ S. K. Khanna \\ Virginia Commonwealth University, snkhanna@saturn.vcu.edu \\ See next page for additional authors
}

Follow this and additional works at: http://scholarscompass.vcu.edu/phys_pubs

Part of the Physics Commons

Cerowski, V., Rao, B. K., Khanna, S. N., et al. Evolution of the electronic structure of Be clusters. The Journal of Chemical Physics 123, 074329 (2005). Copyright (C) 2005 AIP Publishing LLC.

\section{Downloaded from}

http://scholarscompass.vcu.edu/phys_pubs/183

This Article is brought to you for free and open access by the Dept. of Physics at VCU Scholars Compass. It has been accepted for inclusion in Physics Publications by an authorized administrator of VCU Scholars Compass. For more information, please contact libcompass@vcu.edu. 
Authors

V. Cerowski, B. K. Rao, S. K. Khanna, P. Jena, Soh Ishii, Kaoru Ohno, and Yoshiyuki Kawazoe 


\title{
Evolution of the electronic structure of Be clusters
}

\author{
V. Cerowski, B. K. Rao, S. N. Khanna, ${ }^{\text {a) }}$ and P. Jena \\ Physics Department, Virginia Commonwealth University, Richmond, Virginia 23284-2000 \\ Soh Ishii \\ Institute for Materials Research, Tohoku University, 2-1-1 Katahira, Aoba, Sendai 980-8577, Japan \\ Kaoru Ohno \\ Department of Physics, Yokohama National University, 79-5 Tokiwadai, Hodogaya, Yokohama 240-8501, \\ Japan \\ Yoshiyuki Kawazoe \\ Institute for Materials Research, Tohoku University, 2-1-1 Katahira, Aoba, Sendai 980-8577, Japan
}

(Received 27 May 2005; accepted 27 June 2005; published online 25 August 2005)

Using a modified symbiotic genetic algorithm approach and many-body interatomic potential derived from first principles, we have calculated equilibrium geometries and binding energies of the ground-state and low-lying isomers of Be clusters containing up to 41 atoms. Molecular-dynamics study was also carried out to study the frequency of occurrence of the various geometrical isomers as these clusters are annealed during the simulation process. For a selected group of these clusters, higher-energy isomers were more often found than their ground-state structures due to large catchment areas. The accuracy of the above ground-state geometries and their corresponding binding energies were verified by carrying out separate $a b$ initio calculations based on molecular-orbital approach and density-functional theory with generalized gradient approximation for exchange and correlation. The atomic orbitals were represented by a Gaussian $6-311 \mathrm{G}^{* *}$ basis, and the geometry optimization was carried out using the GAUSSIAN 98 code without any symmetry constraint. While the ground-state geometries and their corresponding binding energies obtained from ab initio calculations do not differ much from those obtained using the molecular-dynamics approach, the relative stability of the clusters and the energy gap between the highest occupied and the lowest unoccupied molecular orbitals show significant differences. The energy gaps, calculated using the density-functional theory, show distinct shell closure effects, namely, sharp drops in their values for Be clusters containing 2, 8, 20,34, and 40 electrons. While these features may suggest that small Be clusters behave free-electron-like and, hence, are metallic, the evolution of the structure, binding energies, coordination numbers, and nearest-neighbor distances do not show any sign of convergence towards the bulk value. We also conclude that molecular-dynamics simulation based on many-body interatomic potentials may not always give the correct picture of the evolution of the structure and energetics of clusters although they may serve as a useful tool for obtaining starting geometries by efficiently searching a large part of the phase space. (C) 2005 American Institute of Physics. [DOI: 10.1063/1.2001655]

\section{INTRODUCTION}

The last two decades have witnessed considerable interest in the study of clusters ${ }^{1}$ of metallic and semiconducting elements. One of the most important reasons for this interest is that atomic clusters constitute an intermediate phase of matter between atoms and bulk. Thus, one expects that studies of atomic clusters can illustrate how structure and properties of matter evolve with size and composition-one atom at a time. While much progress has been made in this regard, some fundamental questions still remain to be answered. For example, how many atoms does it take for a metal cluster to behave like a metal?

The difficulty in answering this question underscores two important points: (1) How does one define a metal at the

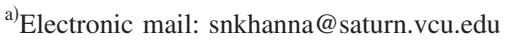

nano- or subnanometer scale, which typically is the size of clusters? If one uses the conventional definition that metals are good conductors of heat and current, atomic clusters are too small for those measurements. (2) One could use electronic signature as a means to study the onset of metallic transition. For example, the electronic band structure of a metal exhibits no gap, i.e., the Fermi energy (the energy of the highest occupied electron) passes through an energy band. In atomic clusters, there are no energy bands, and the electrons occupy discrete molecular orbitals. As clusters grow, the gap between the highest occupied molecular orbital (HOMO) and lowest unoccupied molecular orbital (LUMO) is expected to decrease, and when this gap vanishes, one could argue that the cluster is metallic., ${ }^{2,3}$ Leaving aside the issues of how one measures or calculates these gaps accurately, a nagging question is what if the variation of the HOMO-LUMO gaps is not monotonic? How can a cluster of 
a smaller size be metallic while it ceases to be metallic at a larger size? (3) Another signature of metallic behavior, in at least some systems, is that conduction electrons are nearly free, i.e., they move in a crystal uniformly and their densities are nearly constant throughout the lattice. This is the socalled jellium behavior ${ }^{4}$ where the electrons move in a constant field provided by a uniform distribution of the positiveion cores. Does this mean that if the electronic structure of a cluster can be described by this free-electron jellium model, it can be classified as "metallic" even if the cluster contains only two atoms?

The bottleneck that prevents us from answering these fundamental questions is that electronic and atomic structures of the clusters are intimately linked. There are currently no direct experimental methods that can reveal the geometry of a cluster. The clusters are too small for diffraction probes and too large for spectroscopic techniques. The only way to arrive at the geometry (i.e., atomic arrangements) of a cluster is through theoretical techniques. Even though we ignore for the time being the issue concerning the accuracy of a theoretically derived structure, there are other daunting problems one faces. For example, a cluster can and often may have isomers (i.e., different geometrical forms), which can lie very close in energy. Secondly, it is not clear if experimentally one would always observe the ground-state structure. It is possible that a higher-energy isomer with a large catchment area may be more abundant in a cluster beam than the ground-state structure with a small catchment area. Third, and perhaps the most important factor is that the number of these isomers grows exponentially with cluster size. In a computational procedure it is virtually impossible to search the entire phase space to locate all possible isomers. Thus, one has to be content with mapping the energetically relevant regions of the phase space to determine the isomers that are most likely to be seen in an experiment, and then calculate various measurable properties. If these compare well with experiment, one could be reasonably certain that the geometry and the electronic structure have been properly calculated. One can then see if this cluster possesses electronic signature of metallicity.

As mentioned earlier, these difficulties are the reasons why the above studies have been few and far between. In this paper we address this issue in Be clusters. Using a manybody interatomic potential derived from first principles ${ }^{5}$ and a modified genetic algorithm, ${ }^{6-9}$ we have calculated the geometries and binding energies of hundreds of isomers of $\mathrm{Be}$ clusters containing up to 41 atoms. By a simulated annealing technique, we have also studied the probability that these isomers could be present in a cluster beam. Typically this probability is given by the Boltzmann factor, $e^{-\Delta E / k T}$, namely, if $\Delta E$, energy of the isomer defined with respect to the ground state is large compared to temperature, its probability of being found is small. However, if the potential-energy surface for this isomer is such that there is a large catchment area, its probability of existence may be significant. We have, therefore, searched the energetically relevant regions of phase space and have determined these probabilities. While in most cases the ground-state structure is the most abundant species, there are isolated cases where higher-energy isomers are more abundant than the ground-state structure.

To verify the accuracy of these structures, we have performed independent calculations of the cluster geometries up to 21 atoms using an all-electron density-functional theory (DFT) with generalized gradient approximation for exchange-correlation potential. ${ }^{10}$ The optimized geometries are obtained by using the geometries obtained from molecular-dynamics simulation as starting points and then reoptimizing the final structure without any symmetry constraint as has been done for Ni clusters. ${ }^{11}$ While the geometries and binding energies obtained using the first-principles calculations do not differ significantly from those obtained from molecular-dynamics simulation, the relative stability and the HOMO-LUMO gap differ markedly as these are sensitive to the details of the geometry.

In Sec. II we outline our theoretical procedure. The results are discussed in Sec. III and compared with earlier theoretical calculations, ${ }^{12-16}$ which are limited to the clusters containing up to $21 \mathrm{Be}$ atoms. A summary of our conclusions is given in Sec. IV.

\section{THEORETICAL PROCEDURE}

As pointed out earlier, obtaining the geometrical structure of a cluster is the first step in understanding any of its properties. This is not a simple task as the potential-energy surface may contain a large number of local minima. If any of these minima are protected by a large energy barrier, it is possible that one could arrive at different structures by starting from different initial configurations. As clusters grow, the number of such local minima grows exponentially, and finding the global minimum structure becomes prohibitively difficult. Thus an efficient algorithm is needed to search for the global minimum as well as local minima with large catchment areas. Molecular-dynamics simulation is usually the accepted route for efficiently searching the minima in a potential-energy surface. For this, one needs an interatomic potential, and any error in this choice translates to errors in the computed geometry of the clusters. While a common practice has been to obtain this potential by fitting to some experimental data of the bulk system, its application to atomic clusters is questionable.

In this paper we have adopted the following procedure. (1) We have started with a many-body interatomic potential $\left(V_{n}\right)$ derived for clusters from first principles by BlaistenBarojas and Khanna. ${ }^{5}$ They calculated the total energies of Be clusters containing up to five atoms in various structural forms and then fitted these energies simultaneously to an analytic form containing two-body $V_{2}$ and three-body $V_{3}$ terms. This form was so chosen that as the cluster size increases, three-body interaction term diminishes and one approaches the cohesive energy of the bulk. Using this potential and a modified genetic algorithm, we have studied the potential-energy hypersurface of Be clusters containing up to 41 atoms. For each cluster we have obtained geometries of numerous low-lying isomers by heating the clusters to different temperatures. (2) We used the geometries of the groundstate and those of the low-lying isomers obtained from the 
molecular-dynamics simulation as a starting point in a firstprinciples all-electron calculation based on molecular-orbital formulation and density-functional theory. The geometries of these clusters containing up to 21 atoms were reoptimized without any symmetry constraint. The binding energies, relative stability, and the geometries thus obtained were then compared with those obtained from molecular-dynamics simulation. (3) The HOMO-LUMO gaps were first calculated using the $a b$ initio all-electron molecular-orbital theory on geometries obtained from molecular-dynamics simulation. These were compared with the results of our $a b$ initio calculation where the geometries were further reoptimized without any symmetry constraint. In the following we outline the two separate theoretical procedures.

\section{A. Molecular dynamics using modified genetic algorithm}

Among successfully used molecular-dynamics methods ${ }^{6-9}$ are quenched molecular dynamics, simulated annealing, genetic algorithms (GAs), and "symbiotic" GA as well as GA-inspired Monte Carlo method. Here we use a simple "coordinate-based" GA to find the global and many low-lying local minima of Be clusters. Our goal is to find the geometries of the lowest-energy state and those of the lowlying isomers as a starting point in a more accurate $a b$ initio simulation.

GA is a heuristic approach that mimics the process of natural selection to optimize systems by representing possible states of the system as one-dimensional strings (genes). Optimization then consists of improving the fitness of members of the ensemble of genes (population) by performing operations of crossover, mutation, and selection. Crossover is a binary operation that takes two genes (parents) and replaces them by two other genes (children) obtained by swapping halves of the parents' genes at a randomly chosen position in the gene (crossover point). Mutation consists of small random changes of some members of the population. Selection consists of choosing a subset of the population with the best fitness (lowest potential energy in our case) and replacing the rest of the population by new, usually randomly chosen members. The process is then repeated with the newly obtained population until no significant improvement of the fitness of the population is achieved.

This general scheme of GA can be implemented in many different ways. The GA used in this work starts from an ensemble of randomly chosen clusters with $n$ atoms. Each cluster is then represented by a gene consisting of a sequence of coordinates $x_{i}, y_{i}$, and $z_{i}$ of the $i$ th atom of the cluster, in the order they were generated. The crossover point is chosen between one pair of $3 n$ coordinates. Mutation consists of a small displacement in random direction of one randomly chosen atom of the cluster. The parameters such as the size of the population, the number of configurations with lowest energy kept in the selection, and the number of mutated states are chosen for easier comparison of performances of two algorithms.

The algorithm starts with a population of random clusters of atoms, and the iteration consists of applying crossover-mutation-selection process to a subcluster of atoms within a sphere of radius $R$ around the $i$ th atom of the cluster until the best-found energy has not changed for several consecutive steps. (We found that generally seven steps were enough to guarantee the convergence.) The subcluster is then accepted as part of the cluster only if it further reduces the total energy. This is repeated in sequence for each atom of the fittest cluster with one important difference- $R$ is chosen to be $2.5-4.1 \AA$, going from small to large $n$, which is large enough so that the sphere typically encloses all of the atoms of the cluster. In other words, rather than optimizing subclusters containing about seven atoms of the fittest cluster as in the symbiotic GA (SGA), our algorithm typically optimizes the whole cluster. After applying 200-1000 iterations and repeating the whole process $20-50$ times, the fittest clusters thus obtained are then further "locally" optimized using the conjugate-gradient (CG) method.

The most important advantage of our method over SGA is that it converges toward the global minima for the large number of iterations. This, in turn, is the consequence of doing optimization over the whole cluster rather than subclusters, and, thus, takes into account long-range nature of interatomic forces. SGA, on the other hand, does not necessarily converge toward the global minima because it optimizes subclusters of the whole cluster, whose optimal configurations are usually not beneficial for the entire cluster. The large number of iterations limit of SGA is dominated by subcluster optimizations that do not improve energy of the cluster, and the amount of redundant computation depends sensitively on the degree to which subcluster energy is optimized-better is this optimization done, more likely is that the optimized subcluster will not improve the energy of the entire cluster.

If we furthermore notice that the three-body term of the potential requires $\mathrm{O}\left(n^{3}\right)$ evaluations of $V_{3}$, the total time that SGA takes for evaluation will be dominated by the number $M$ of CG minimizations done. Assuming that $M$ is about the same as in the case of the Lennard-Jones potential, $M=5$ $\times 10^{4}$, it can be estimated that our algorithm has available $M \approx 500$ to perform the same number of operations. In practice, on the other hand, it seems that $M \approx 300$ used in this work is sufficient to locate the global and low-lying local minima.

Compared with the methods of Ref. 17, we notice that these employ both "cutting" of clusters using planes and local Monte Carlo (MC) moves at a fixed temperature. Qualitatively, the crossover operation employed here contains these cutting operations as well as a considerably larger set of operations of combining two clusters into one that cannot be represented through few cutting operations, while the mutation produces local moves. For these reasons, we do not expect that these methods can outperform ours.

In order to further check whether global minima are reached in the simulations, we carried out additional five simulations, each $10^{4}$ steps long, for $n \leqslant 25$ and without further CG minimization, and the best energy found was less than $0.1 \%$ higher from the already found ground state. 


\section{B. Density-functional calculations}

The total energy of a cluster with a given geometry is calculated using DFT and generalized gradient approximation (GGA) for exchange-correlation potential. We have used the BPW91 (Ref. 18) form for the GGA which incorporates Becke's exchange and Perdew-Wang's prescription for correlation. The forces at each atomic site are calculated from the gradient of the total energies. The atoms are then moved to a new location following the path of steepest descent until the forces at every atomic site vanish. No symmetry was enforced during this optimization procedure. We have used the GAUSSIAN $98 \operatorname{code}^{19}$ for these calculations. The atomic orbitals were given by 6311-G** Gaussian basis. The binding energies per atom $E_{b}$ and the energy gain $\Delta E$ in adding an atom to an existing cluster are calculated from the total energy $E(n)$ using the following equations:

$$
\begin{aligned}
& E_{b}=-\left[E(n)-n E_{0}\right] / n, \\
& \Delta E=-[E(n+1)-E(n)] .
\end{aligned}
$$

Here $n$ is the number of atoms in the cluster and $E_{0}$ is the energy of the atom. The vertical ionization potential (I.P.) was calculated as the difference in energy between the neutral and positively charged clusters at the neutral geometry, namely,

$$
\text { I.P. }=-\left[E(n)-E^{+}(n)\right] .
$$

\section{RESULTS AND DISCUSSIONS}

The geometries of Be clusters containing up to 21 atoms have been studied previously using different theoretical techniques. ${ }^{12-16}$ While quantum-chemical methods have been used for Be clusters containing less than eight atoms, density-functional theory combined with Car-Parrinello molecular-dynamics simulation has been used by Kawai and Weare ${ }^{12}$ and by Wang et al. ${ }^{16}$ to calculate the geometry, binding energy, and HOMO-LUMO gap of Be clusters up to 21 atoms. The authors conclude that Be clusters exhibit a metallic behavior with as small as seven atoms because of significant $p$ character of the molecular orbitals. In addition, Kawai and Weare claim that Be clusters containing as small as 11 atoms can be regarded as a fragment of the hcp lattice. While much of our cluster geometries and structural properties agree with these authors, our conclusions are different. For example, we see icosahedric growth pattern up to the largest cluster we have studied, namely, 41 atoms. This is not a signature of bulk behavior. In addition, we have computed the ionization potentials of these clusters and compared these with the systematic variation in the HOMO levels and commented on the validity of the Koopmans theorem.

We present our results in two different steps: (1) We discuss the evolution of the geometry, nearest-neighbor distance, coordination number, and binding energy per atom calculated from the ground-state structure of clusters as obtained from the molecular-dynamics study. We also discuss the existence of isomers for each of the 41 clusters. (2) We next present the ground-state geometries as obtained from the molecular-orbital approach within the DFT up to 21 at- oms and compare their properties with those obtained from the molecular-dynamics study mentioned above. We also compare the relative stability and the evolution of the HOMO-LUMO gaps calculated from the molecular-orbital approach and molecular-dynamics geometries up to 21 atoms. Finally, we present results on the evolution of the ionization potentials and compare them with the variations based on Koopmans theorem.

\section{A. Results based on molecular-dynamics study}

In Fig. 1 we plot the relative energies of isomers lying within $0.075 \mathrm{eV} /$ at. of the ground-state energy for clusters containing up to 41 atoms. We note that the number of isomers increases dramatically as the cluster size increases. Not all the parts of configuration space are equally accessible in the course of a simulation. In Fig. 2 we plot the density of occurrences of the isomers. This is determined by calculating how many times each of the low-lying minima has been found. Since our algorithms converge toward the global minima in the limit of large number of iterations (when the corresponding distributions are $\delta$ functions at the groundstate energy), distributions in Fig. 2 are indicative of the algorithm dynamics on a short time scale which, in turn, are influenced by the size of the "catchment areas" of different local minima. Low density of occurrence of a particular global minimum then suggests that the cluster configuration corresponding to the global minima will be suppressed in experiments that do not produce highly equilibrated distributions of cluster, and therefore, the cluster configurations corresponding to the higher-energy isomers with high number of occurrences are expected to be much more abundant and, therefore, relevant for the correct interpretation of experimental findings.

Depending on the properties of the distribution of occurrences, we tentatively notice the following three classes: (i) The largest class corresponds to clusters where the ground state was most frequently observed. These correspond to $n$ $=2-6,8,10-13,15,16,19,20,22-26,28,30$, and 35. (ii) Clusters where there is a single local minimum that is significantly more frequently obtained than all other minima, including the lowest one, occur at $n=7,14,18,21,31,32$, and 34 , and (iii) clusters where there are several local minima approximately equally frequently obtained are at $n$ $=17,33$, and 36-41.

In Fig. 3 we plot the ground-state geometries of Be clusters containing up to 41 atoms. The clusters assume threedimensional form while containing as few as four atoms. A pentagonal arrangement of atoms, which is the seed for icosahedric growth, first appears in the $\mathrm{Be}_{7}$ cluster. $\mathrm{Be}_{13}$ is the first complete icosahedric cluster. As cluster size increases, one notes that the icosahedric structure remains as the building block. The structure of $\mathrm{Be}_{19}$, for example, is a double icosahedron. Even the largest cluster studied here does not mimic the hep crystal structure of Be. Thus, one can say that as far as the atomic structure of Be is concerned, the 41 atom cluster does not represent a fragment of the bulk crystal. It is worth noting that the icosahedric growth pattern has also been observed for $\mathrm{Na}$ clusters containing up to 59 


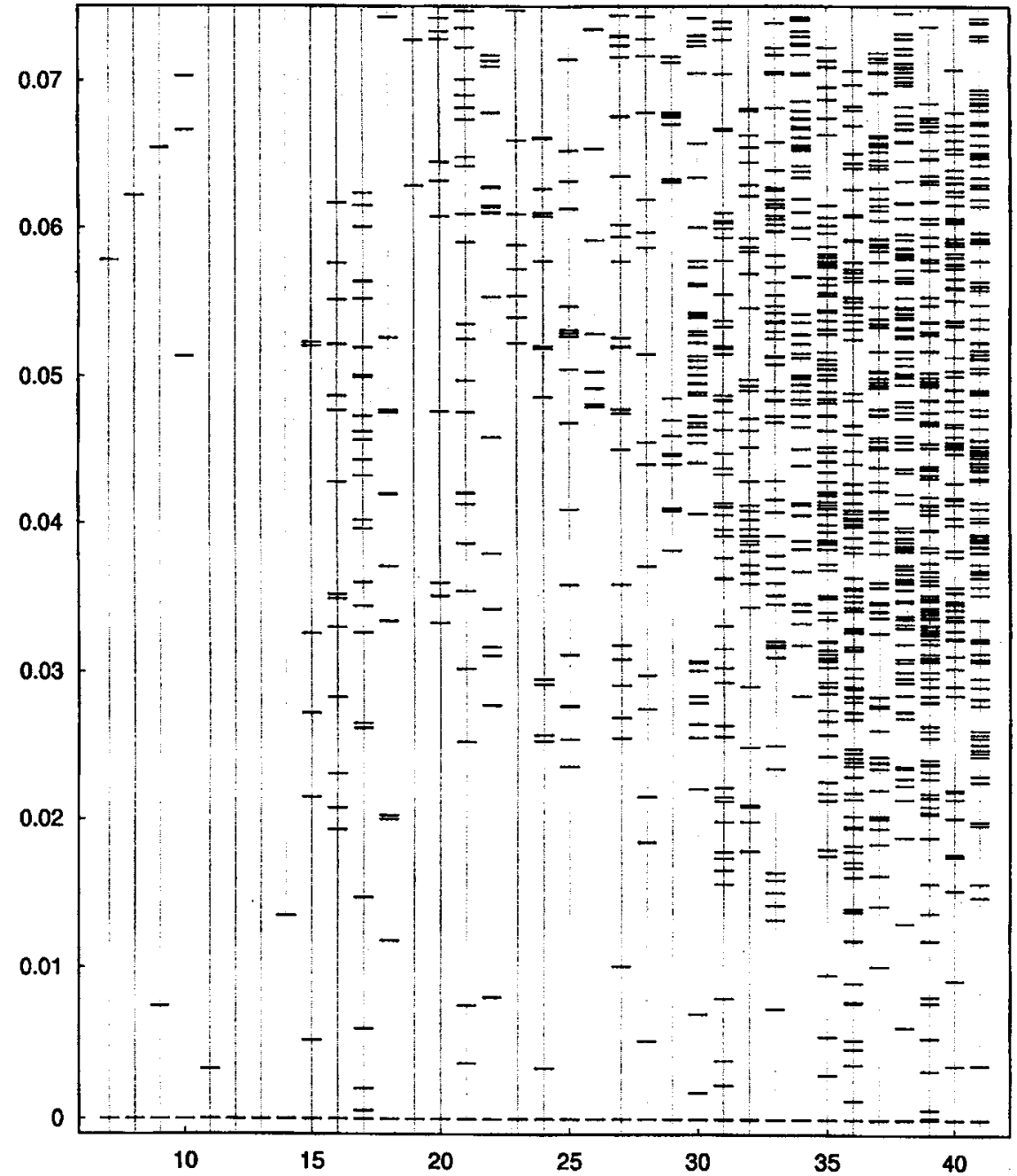

FIG. 1. Energies of low-lying minima relative to the ground-state energy per atom $\left(E_{n}^{i}-E_{n}^{0}\right) / n$ (in eV) vs the cluster size $n$. atoms by Kummel et al. ${ }^{20}$ In general, as we will show in the following, the electronic shell effects and the icosahedric growth work hand-in-hand for metal clusters.

In Fig. 4(a) we plot the evolution of the nearest-neighbor distance calculated using Eqs. (4) and (5). This is calculated by summing up all the bond lengths $-R_{i j}$ in a cluster that is within $20 \%$ of the bulk nearest-neighbor distance, $R_{0}$ and dividing the result by the number of such bonds, $N$, namely,

$$
\begin{aligned}
& R=\Sigma R_{i j} / N . \\
& R_{i j} / R_{0} \leqslant 1.20 .
\end{aligned}
$$

Note that the nearest-neighbor distance increases monotonically with cluster size. Although the incremental change in these distances diminishes with cluster size, there is no indication that the values have saturated. Even for the largest cluster studied, the nearest-neighbor distance is about $5 \%$ larger than the bulk value of $2.22 \AA$. This is in contrast with most metals where the average nearest distance in clusters is less than the bulk value. The anomaly in the Be clusters can be understood by comparing the results with interplanar relaxation at the (0001) surface of Be (Refs. 21 and 22) where the surface layer was found to relax outward by $5.8 \% .^{22}$ This anomalous relaxation has been attributed to the closed electronic shell $\left(1 s^{2} 2 s^{2}\right)$ of the Be atom. The surface atoms, due to low coordination, behave more like the closed-shell at- oms. The bulk atoms, on the other hand, are more metallic due to strong hybridization between the $s$ and $p$ states. Note that in the Be clusters studied here most of these atoms are surface atoms. One can, therefore, conclude that the electronic structure of Be clusters containing up to 41 atoms has not reached the bulk limit. This is contrary to the conclusion made by Kawai and Weare. ${ }^{12}$

In Fig. 4(b) we plot the coordination number $C_{N}$ as a function of cluster size. This is calculated by counting the number of nearest-neighbor atoms $N_{i j}$ lying within a distance of $R_{i j}$ from each atom $i$ in the cluster and dividing by the number of atoms $n$ in the cluster.

$$
C_{N}=\Sigma N_{i j} / n
$$

Note that the coordination numbers evolve rather slowly toward the bulk value of 12 as most of the atoms are surface atoms.

In Fig. 5(a) we plot the binding energy per atom as a function of size. The energies increase rapidly up to six atoms and then slowly for larger clusters. But the energy of the largest cluster studied here is significantly lower than the bulk cohesive energy of $3.32 \mathrm{eV}$. To determine the accuracy of the binding energies calculated from the many-body interatomic potential, we have recalculated these using the DFTbased molecular-orbital approach with the geometries ob- 


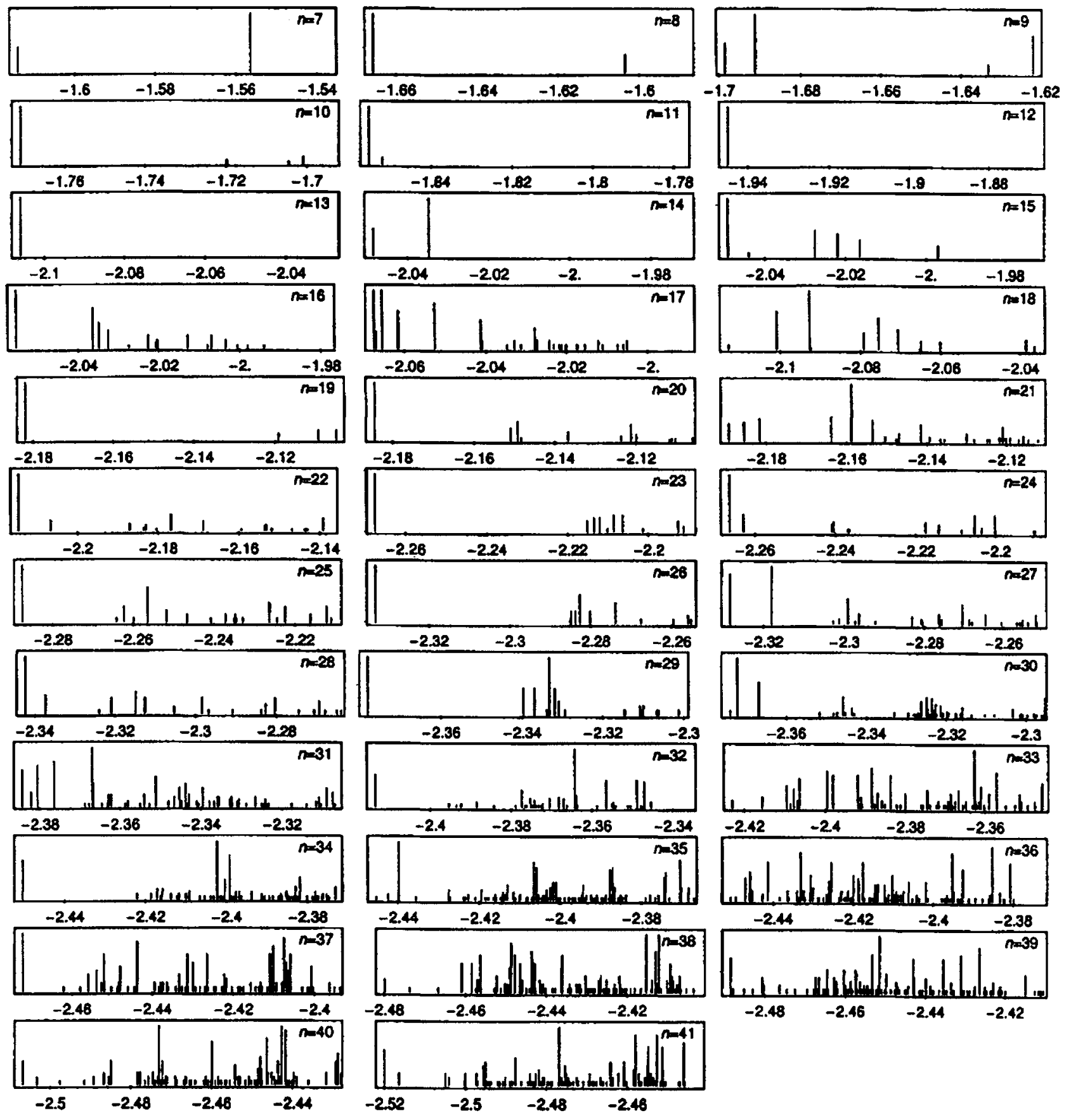

FIG. 2. Distribution of density of occurrences of different isomers corresponding to the low-lying minima. The vertical axis scales the number of times a given isomer occurred during the simulation process.

tained in GA simulation. These energies are given in Table I. These two energies agree well with each other for most clusters within an accuracy of $0.07 \mathrm{eV}$. The exceptions are for $\mathrm{Be}_{4}, \mathrm{Be}_{6}, \mathrm{Be}_{7}$, and $\mathrm{Be}_{13}$ where the GA-computed binding energies differ from the self-consistent-field (SCF)-linear combination of atomic-orbital (LCAO)-molecular-orbital (MO) values with GA geometries by $-0.12,-0.38,0.14$, and $0.23 \mathrm{eV}$, respectively.

To demonstrate more clearly the relative stability of the clusters, we have calculated the energy $\Delta E$ gained in adding a single atom to an existing cluster [Eq. (2)]. In Fig. 6(a) we plot $\Delta E$ 's obtained from our all-electron calculations based on geometries obtained from the GA simulation. The $\Delta E$ values obtained from our fully reoptimized geometries using DFT and the GAUSSIAN98 code are given in Fig. 6(b). We first note that while the binding energy per atom $E_{b}$ plotted in Fig. 5 at different levels of theory does not appear to be very different from each other, the values of $\Delta E$ in Fig. 6 show marked differences. For example, the results in Fig. 6(b) based on the molecular-orbital approach yield sharp drop at $n=4,10,17$, and 20 . These correspond to the clusters containing $8,20,34$, and 40 electrons which are precisely the values at which shell closings occur in a jellium model. The $\Delta E$ 's obtained from the geometries based on GA simulation in Fig. 6(a) do not exhibit such a clear pattern. This indicates 


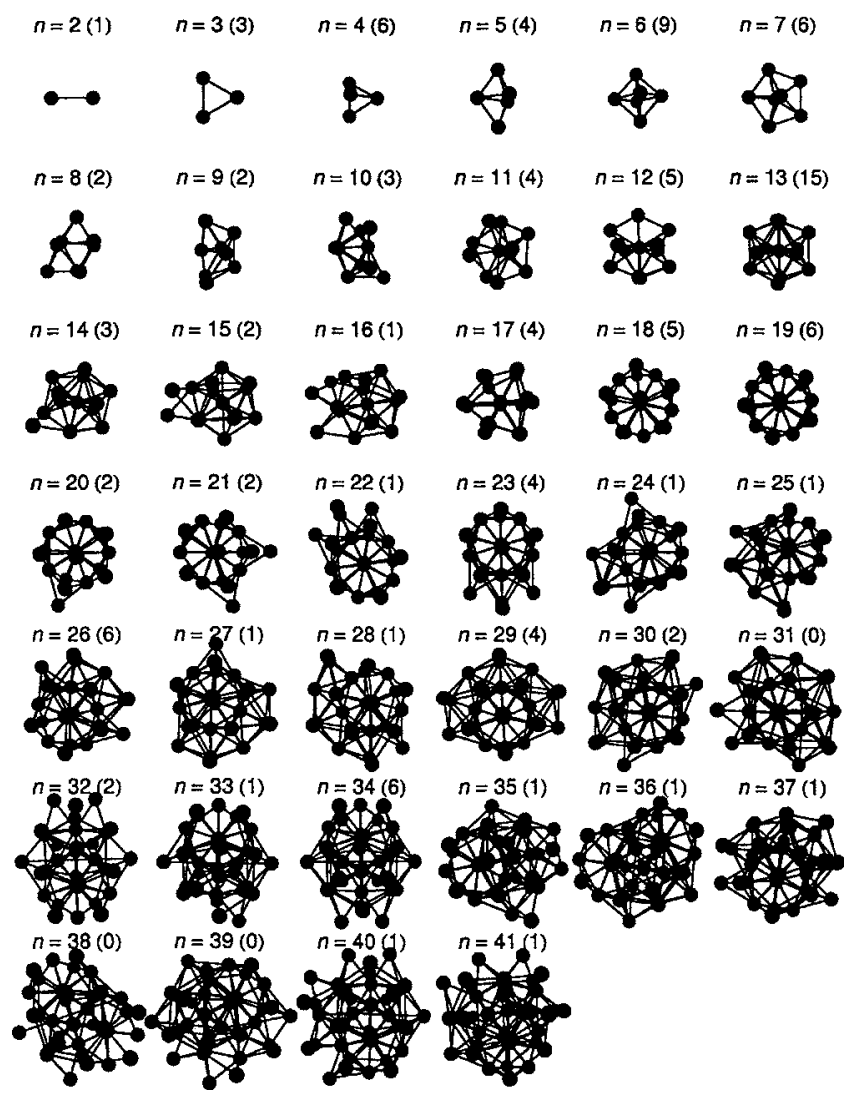

FIG. 3. Geometries corresponding to the ground state of clusters obtained in the GA simulation. The number of reflection planes for each cluster is given in parentheses. Clusters are plotted such that the symmetry as well as "atomic shell" structures is clearly visible.

that even the most comprehensive molecular-dynamics simulation can fail to account for the correct physics if the manybody potential does not take into account relaxation of electron eigenfunctions with changing geometry. We will show
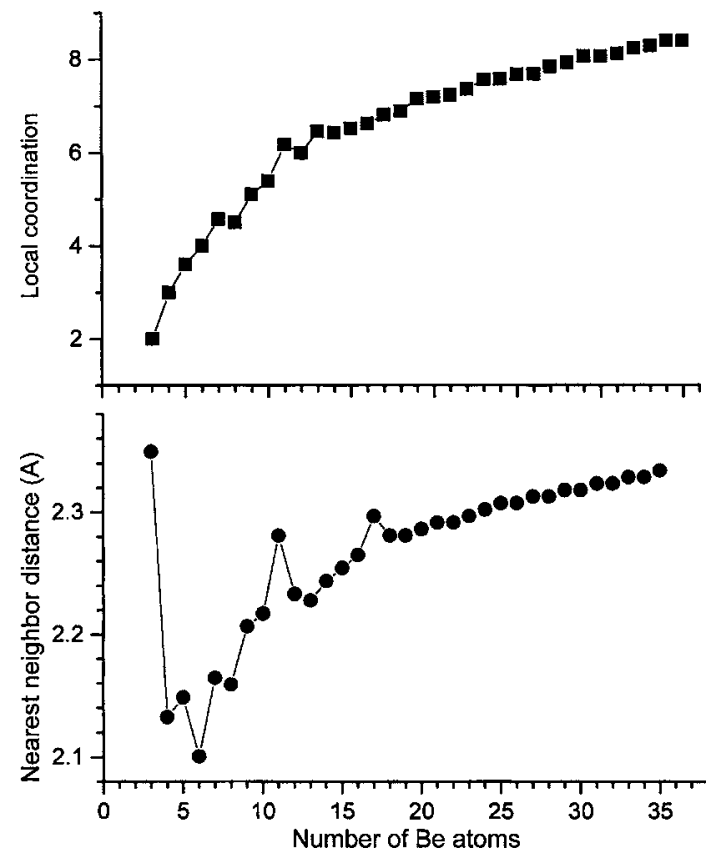

FIG. 4. (a) Nearest-neighbor distance and (b) coordination number as a function of size.

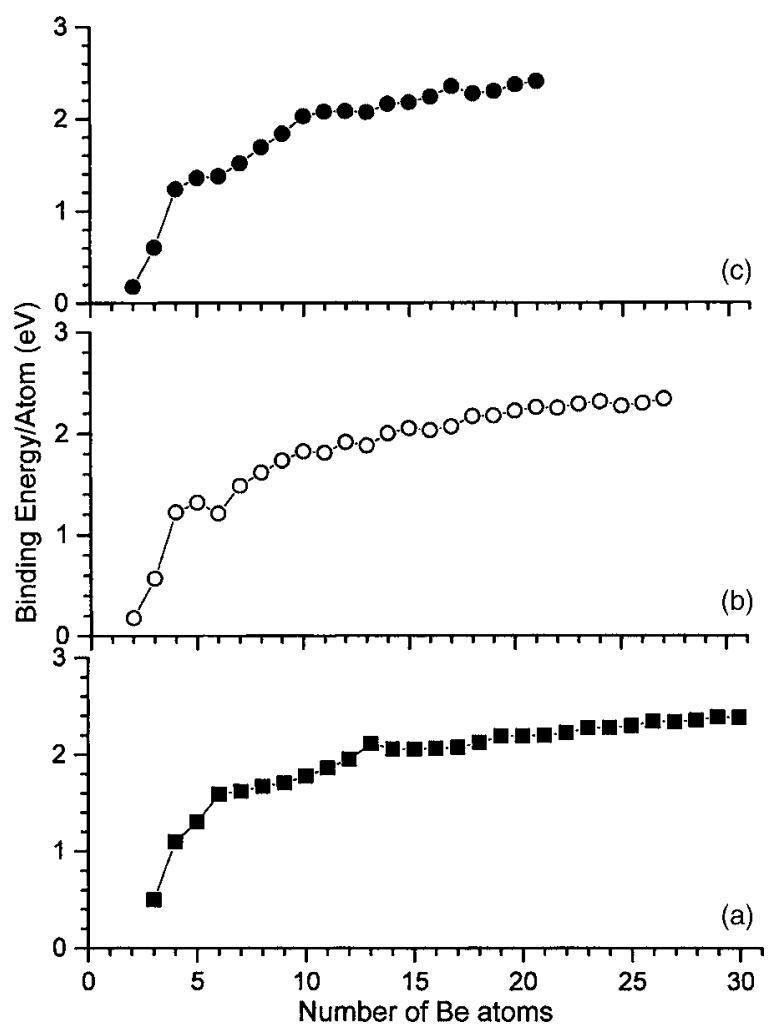

FIG. 5. Binding energies per atom as a function of size. (a) Results from GA calculation. (b) SCF-LCAO-MO results based on GA geometries. (c) SCFLCAO-MO results with reoptimized geometries.

later that the same difficulty arises in accounting for the HOMO-LUMO gaps.

\section{B. Results based on molecular-orbital calculations}

Molecular-dynamics approach discussed above is capable of yielding geometrical structures and their corre-

TABLE I. The binding energy per atom (eV) evaluated from the GA (column 2) simulations, SCF-LCAO-MO calculations with geometries as in GA simulation (column 3), and SCF-LCAO-MO calculations on optimized geometries (column 4).

\begin{tabular}{cccc}
\hline \hline$n$ & GA & $\begin{array}{c}\text { SCF-LCAO-MO with } \\
\text { GA geometries }\end{array}$ & $\begin{array}{c}\text { SCF-LCAO-MO optimized } \\
\text { geometries }\end{array}$ \\
\hline 3 & 0.50 & 0.57 & 0.61 \\
4 & 1.10 & 1.22 & 1.24 \\
5 & 1.30 & 1.32 & 1.36 \\
6 & 1.59 & 1.21 & 1.38 \\
7 & 1.62 & 1.48 & 1.52 \\
8 & 1.67 & 1.61 & 1.69 \\
9 & 1.70 & 1.74 & 1.84 \\
10 & 1.78 & 1.82 & 2.03 \\
11 & 1.86 & 1.81 & 2.08 \\
12 & 1.95 & 1.92 & 2.08 \\
13 & 2.11 & 1.88 & 2.08 \\
14 & 2.05 & 2.00 & 2.16 \\
15 & 2.06 & 2.05 & 2.18 \\
16 & 2.06 & 2.03 & 2.24 \\
17 & 2.07 & 2.07 & 2.35 \\
18 & 2.12 & 2.17 & 2.27 \\
19 & 2.19 & 2.18 & 2.30 \\
20 & 2.19 & 2.22 & 2.37 \\
21 & 2.20 & 2.26 & 2.41 \\
\hline \hline
\end{tabular}




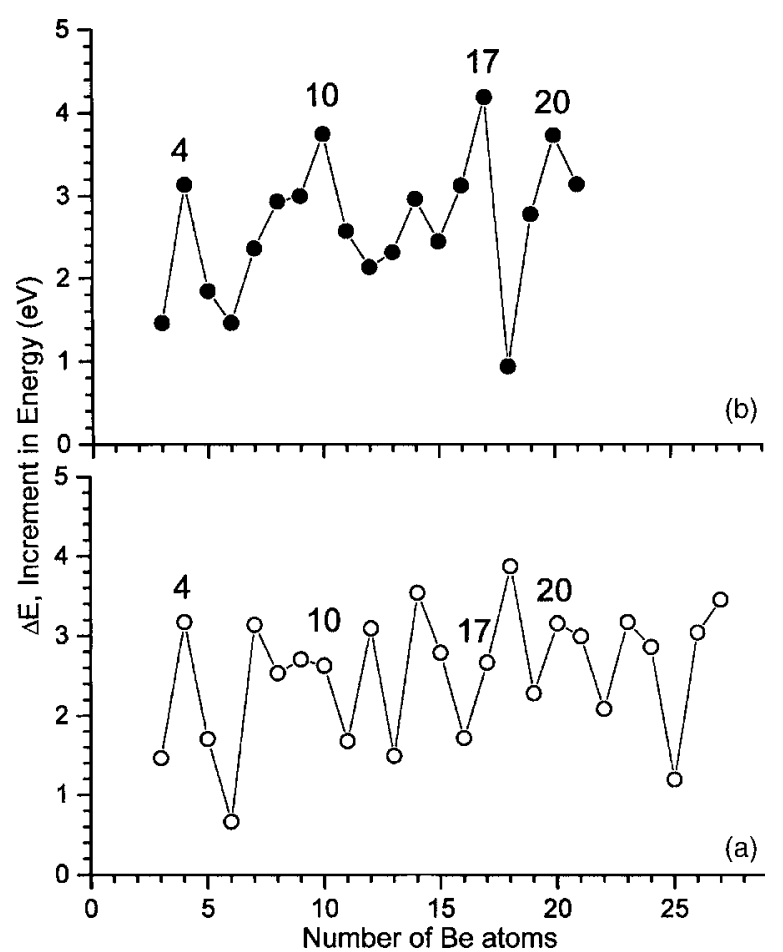

FIG. 6. $\Delta E$ increment energy in adding an atom to a preexisting cluster. (a) SCF-LCAO-MO results based on GA geometries. (b) SCF-LCAO-MO results with reoptimized geometries.

sponding binding energies but provides no information on the electronic structure such as electron charge distribution, electron energy-level spectrum, and, hence, the HOMOLUMO gap. To obtain such information, one has to use the molecular-orbital calculations.

We have, therefore, reoptimized the geometries of $\mathrm{Be}$ clusters up to 21 atoms starting with the GA ground-state geometries and those of low-lying isomers as starting configurations. No symmetry constraint was imposed. Larger clusters were not attempted due to significant computational costs. In Fig. 7 we compare the geometries obtained from our ab initio calculation with those based on the GA simulation. Note that apart from a small difference in bond distances, geometries of most clusters agree with each other. The exceptions are for $n=11,12,14$, and 17 . We will show that in spite of these small differences, the calculated HOMO-LUMO gaps are rather sensitive, as we have noticed to be the case for relative stability in Fig. 6 .

We next discuss the evolution of the binding energies per atom. The results are plotted in Fig. 5(c) as a function of size. Although the trend in the variation of $E_{b}$ is similar to that in Figs. 5(a) and 5(b), significant differences are noticed. For example, the binding energies of $\mathrm{Be}_{5}$ and $\mathrm{Be}_{6}$ clusters are nearly the same in the molecular-orbital approach while in the GA approach, $\mathrm{Be}_{6}$ binding energy is significantly larger than that of $\mathrm{Be}_{5}$. Similarly $\mathrm{Be}_{13}$ is found to be locally more stable than the $\mathrm{Be}_{12}$ or $\mathrm{Be}_{14}$ in the GA approach. No such behavior is seen from the molecular-orbital calculations. These differences are accentuated in the plot of $\Delta E$ in Fig. 6(b). Note that in the MO calculations, we do find sharp drops at $n=4,10,17$, and 20 as expected from shell closure effects. (a)
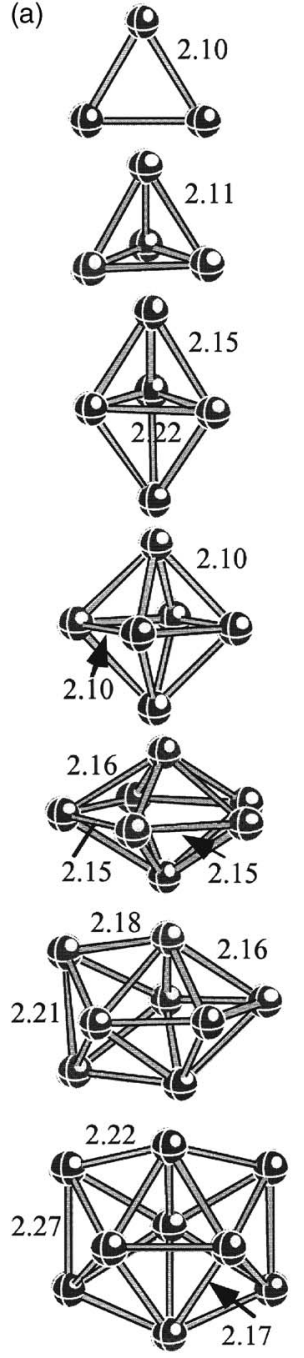

(b)
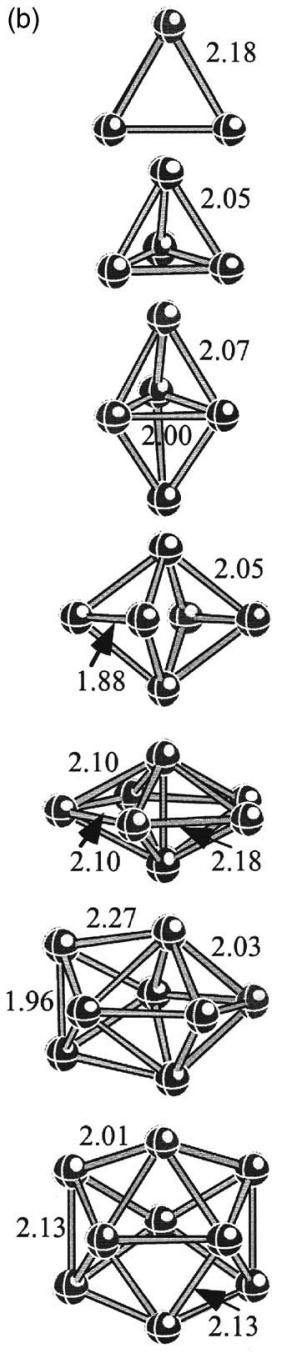

FIG. 7. Comparison of geometries obtained from (a) GA method and (b) reoptimized at the SCF-LCAO-MO level of theory.

The striking difference, however, appears in the HOMOLUMO plot in Fig. 8. In Fig. 8(a) we plot the HOMOLUMO gaps calculated using the ground-state geometries obtained in the GA approach. We note that there are sharp drops in these gaps at $n=1$ and 4 which correspond to 2 and 8 valence electrons, respectively. This is consistent with shell closure effects. But we do not observe any such sharp drops at $n=10,17$, or 20 which contain 20,34, and 40 electrons, respectively. These correspond to shell closures in a jellium model, and experimental data on $\mathrm{Mg}$ clusters ${ }^{2,3}$ do exhibit drops in the HOMO-LUMO gaps that are consistent with the jellium model. Figure 8(b) presenting data from optimized MO calculations shows the drops at all of these sizes, i.e., at $n=1,4,10,17$, and 20 , corresponding to magic clusters.

In Fig. 9(a) we plot the vertical ionization potentials. Note that these decrease with size with significant drops at $n=1,4,10,17$, and 20 which are consistent with shell structure effects as well as with large HOMO-LUMO gaps in Fig. 8 . The vertical ionization potentials are often linked to the HOMO energy level through the Koopman's theorem. This theorem which is based on Hartree-Fock approximation states that the energy difference between two systems containing $N$ and $N-1$ electrons is equal to the energy required 
(a)
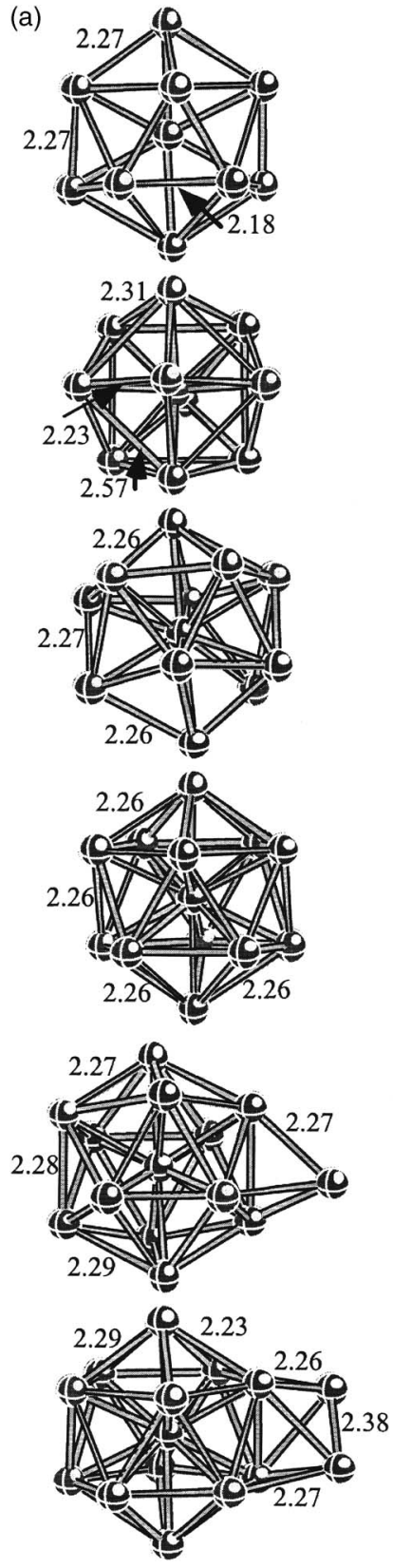

(b)
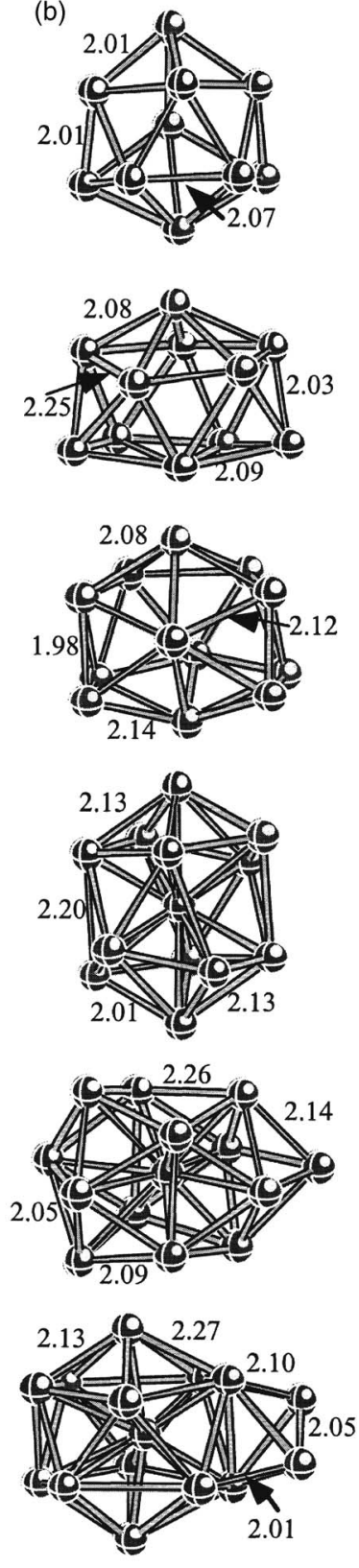

FIG. 7. (Continued)

to remove an electron from state, say $j$, to state $i$, namely, $\Delta E=E_{i}-E_{j}$, where $E_{i}\left(E_{j}\right)$ is the energy of state $i(j)$. The validity of the Koopman's theorem requires that the oneelectron wave functions remain unchanged during this process. The questions regarding the validity of Koopman's theorem in clusters or within the framework of densityfunctional theory notwithstanding, we plot in Fig. 9(b) the negative of the HOMO levels obtained from our MO study as a function of cluster size. We note that the HOMO levels reproduce the variation with size of the ionization potential remarkably well. For example, the sharp drops in the HOMO values at $n=1,4,10,17$, and 20 are as we have noted in Fig. 9(a). However, the magnitude of the HOMO levels is consistently smaller than the vertical ionization potential by about $2 \mathrm{eV}$. To demonstrate this clearly, we have shifted the HOMO values in Fig. 9(b) upwardly by $2.1 \mathrm{eV}$ and plotted (a)
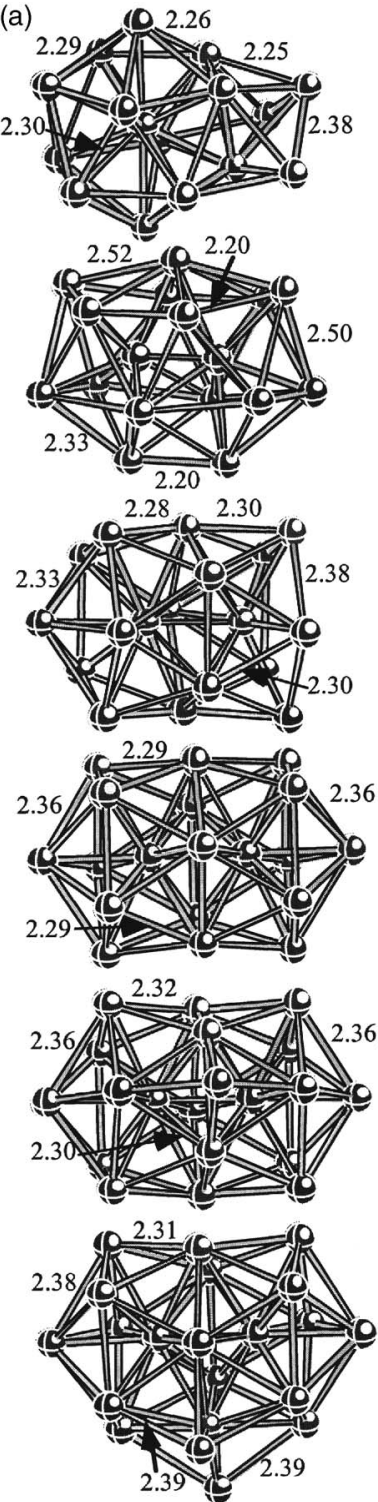

(b) $204 \cdot 0.2 .32$
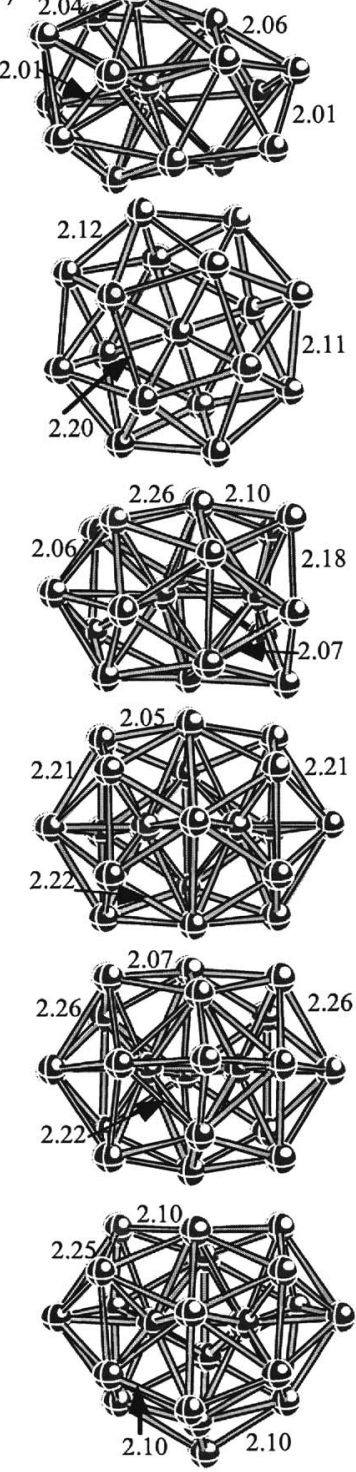

FIG. 7. (Continued)

these results as open circles in Fig. 9(a). Note that the agreement is quite good in the range of $4<n \leqslant 12$. The HOMO values are underestimated in $n \leqslant 4$ clusters and overestimated in $n>13$ clusters. Thus, the HOMO levels obtained in the density-functional theory can account for the trend in the ionization potential, although not its magnitude.

\section{SUMMARY AND CONCLUSIONS}

Equilibrium geometries of the ground-state and lowlying isomers of Be clusters containing up to 41 atoms were obtained from a rigorous search of the available phase space by using molecular-dynamics simulation based on a coordinate-based genetic algorithm and many-body interatomic potential. The evolution of the structures bears the signature of an icosahedric growth. The structural parameters such as equilibrium geometries, the average nearest-neighbor distances, and the coordination numbers do not resemble the bulk. Similarly, the binding energy evolves slowly and no evidence of bulklike behavior is found. 


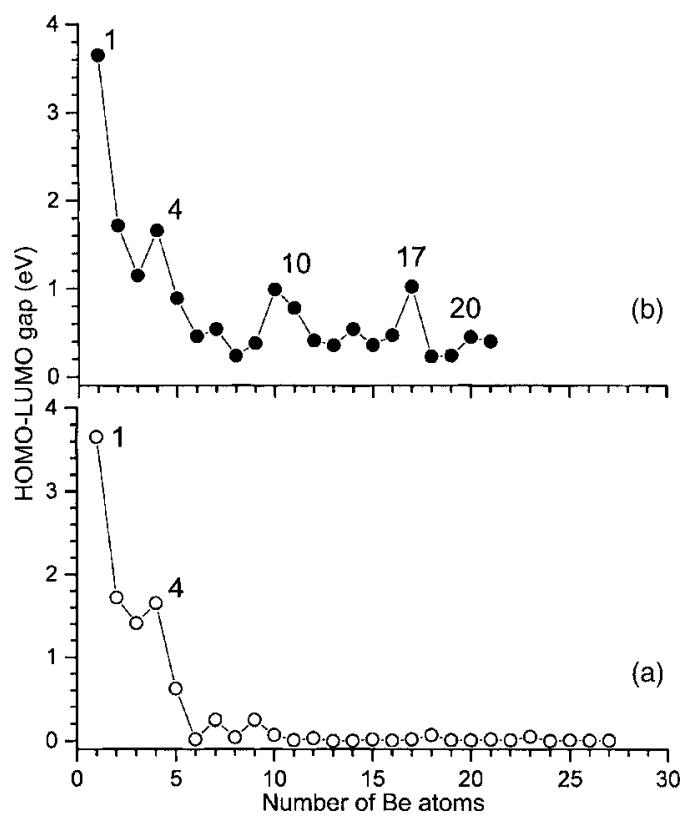

FIG. 8. HOMO-LUMO gaps based on (a) GA geometries and (b) geometries reoptimized at the SCF-LCAO-MO level of theory.

Calculations were repeated with the MO formalism within the framework of density-functional theory for clusters containing up to 21 atoms. While the optimized groundstate geometries at this level of theory do not differ much
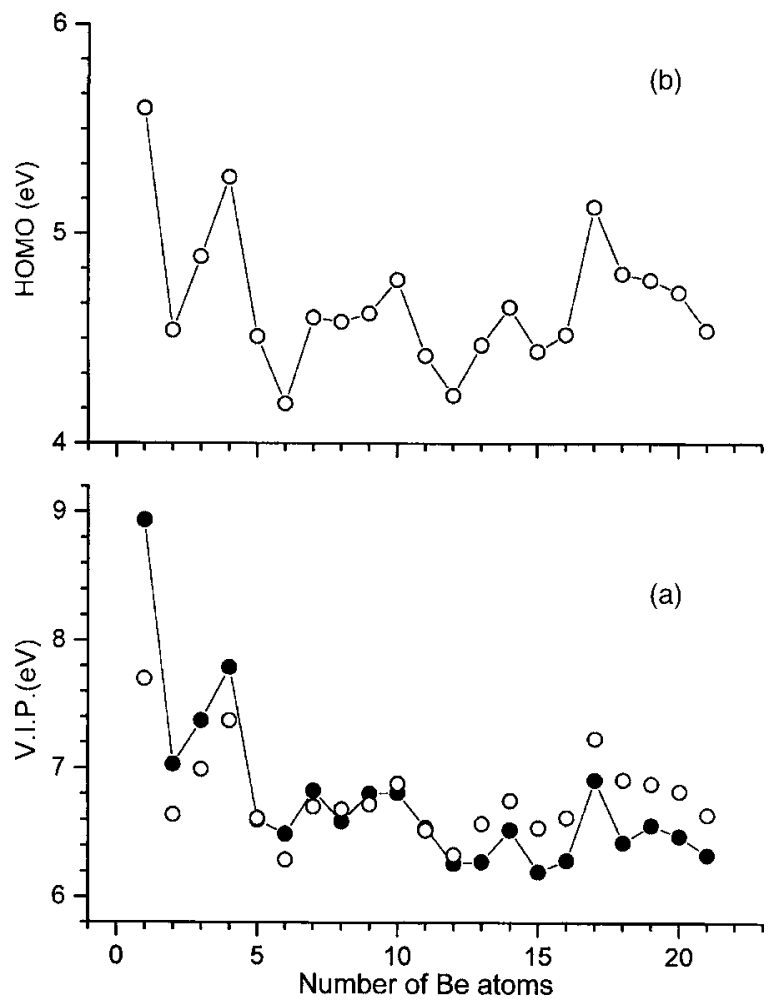

FIG. 9. (a) Vertical ionization potential and (b) HOMO-level calculated using the SCF-LCAO-MO approach. The open circles in (a) are obtained by shifting the HOMO levels upward by $2.1 \mathrm{eV}$. from those based on GA approach, the relative stability and the electronic properties such as HOMO-LUMO gaps are found to be very sensitive to cluster geometry. Distinct shell structure effects are noticed from the calculated stability, HOMO-LUMO gaps, and ionization potentials. The latter shows characteristic drops at $n=1,4,10,17$, and 20 atoms which correspond to $2,8,20,34$, and 40 electron systems. Although small Be clusters show features in agreement with the jellium model, their significant HOMO-LUMO gaps do not permit them to be classified as metallic. We also conclude that while molecular-dynamics simulation based on interatomic potential may be useful in searching the available phase space more efficiently for the geometries of the ground-state and low-lying isomers, a quantitative and in some cases even qualitative understanding of the evolution of the structure and binding energies of clusters may require a first-principles approach.

\section{ACKNOWLEDGMENT}

The work was supported in part by a grant (DEFG0296ER45579) from the Department of Energy.

${ }^{1}$ Cluster Assembled Materials, edited by K. Sattler (TransTech, Switzerland, 1996); Small Particles and Inorganic Clusters, edited by H. H. Anderson (Springer, New York, 1997); Atomic Clusters and Nanoparticles, Les Houches Session LXXIII, edited by C. Guet (Springer, Heidelberg, 2001).

${ }^{2}$ O. C. Thomas, W. J. Zheng, S. J. Xu, and K. H. Bowen, Phys. Rev. Lett. 89, 213403 (2002).

${ }^{3}$ P. H. Aciolo and J. Jellinek, Phys. Rev. Lett. 89, 213402 (2002).

${ }^{4}$ W. D. Knight, K. Clemenger, W. A. de Heer, W. A. Saunders, M. Y. Chou, and M. L. Cohen, Phys. Rev. Lett. 52, 2141 (1984); W. Ekardt, Phys. Rev. B 29, 1558 (1984).

${ }^{5}$ E. Blaisten-Barojas and S. N. Khanna, Phys. Rev. Lett. 61, 1477 (1988).

${ }^{6}$ D. E. Goldberg, Genetic Algorithms in Search, Optimization, and Machine Learning (Addison-Wesley, Massachusetts, 1989).

${ }^{7}$ D. M. Deaven and K. M. Ho, Phys. Rev. Lett. 75, 288 (1995).

${ }^{8}$ D. M. Deaven, N. Tit, J. R. Morris, and K. M. Ho, Chem. Phys. Lett. 256, 195 (1996).

${ }^{9}$ K. Michaelian, Chem. Phys. Lett. 293, 202 (1998).

${ }^{10}$ R. G. Parr and W. Yang, Density Functional Theory of Atoms and Molecules (Oxford University Press, London, 1989).

${ }^{11}$ S. K. Nayak, S. N. Khanna, B. K. Rao, and P. Jena, J. Phys.: Condens. Matter 10, 10853 (1998).

${ }^{12}$ R. Kawai and J. H. Weare, Phys. Rev. Lett. 65, 80 (1990).

${ }^{13}$ P. V. Sudhakar and K. Lammertsma, J. Chem. Phys. 99, 7929 (1993).

${ }^{14}$ A. M. Kolchin and R. W. Hall, J. Chem. Phys. 113, 4083 (2000).

${ }^{15}$ M. K. Beyer, L. A. Kaledin, A. L. Kaledyn, M. C. Heaven, and V. E. Bondybey, Chem. Phys. 262, 15 (2000).

${ }^{16}$ J. Wang, G. Wang, and J. Zhao, J. Phys.: Condens. Matter 13, L753 (2001)

${ }^{17}$ I. Rata, A. A. Shvartsburg, M. Horoi, T. Frauenheim, K. W. Michael Siu, and K. A. Jackson, Phys. Rev. Lett. 85, 546 (2000).

${ }^{18}$ J. P. Perdew, J. A. Chevary, S. H. Vosko, K. A. Jackson, M. R. Pederson, D. J. Singh, and C. Fiolhais, Phys. Rev. B 46, 6671 (1992).

${ }^{19}$ M. J. Frisch, G. W. Trucks, H. B. Schlegel et al., GAUSSIAN 98, Revision A.7, Gaussian, Inc., Pittsburgh, PA, 1998.

${ }^{20}$ S. Kummel, M. Brack, and P. G. Reinhard, Phys. Rev. B 62, 7602 (2000).

${ }^{21}$ A. Antonelli, S. N. Khanna, and P. Jena, Surf. Sci. Lett. 289, L614 (1993).

${ }^{22}$ H. L. Davis, J. B. Hannon, K. B. Ray, and E. W. Plummer, Phys. Rev. Lett. 68, 2632 (1992). 